\title{
Hospital blood bank laboratory data processing
}

\section{system}

\author{
IR CLARK, J PAREKH, MARGARET PETERS, IDO FREW, ${ }^{*}$ RN IBBOTSON†
}

From the Department of Clinical Chemistry, Wolfson Research Laboratories, Queen Elizabeth Medical Centre, Birmingham, the *Department of Haematology, Good Hope Hospital, Sutton Coldfield, West

Midlands, and the †West Midlands Regional Blood Transfusion Service, Edgbaston, Birmingham

SUMMARY A data processing system designed to improve the management and usage of blood and blood products has been developed as a pilot for general application throughout the West Midlands Regional Health Authority.

The package provides for the routine clerical, patient record, and stock management aspects of a hospital blood bank laboratory. It can be tailored to the individual requirements of different laboratories and may be implemented in a variety of ways on a family of computer systems of differing power and capacity, thus accommodating widely varying workloads and archiving requirements. Bar code readers are included in the system design, and may be used for data input where standard Codabar labels are affixed to blood products by blood transfusion centres.

The system has been installed in one hospital laboratory and preliminary results indicate a high degree of acceptability at all levels. Further refinement of the software to meet minor deficiencies and the requirements of the recent DHSS Health Circular BLD/2/13 are currently being specified.

The need for improvements in the management and usage of blood products has generated interest in the introduction of data processing techniques to hospital blood bank laboratories. In contrast to other branches of pathology where data processing techniques are, in the main, reasonably well established, hospital blood banking has not been given sufficient attention. This omission in a branch of pathology where mistakes in clerical or analytical procedures, or both, can result in the death of the patient is attributable to a number of factors. Relatively low request rates compared with other branches of pathology probably mitigated against development, since clinical laboratory data processing has been largely associated with higher throughput laboratories where greater equipment costs are a more recognised norm. The availability of lower cost computer hardware obviates this problem. In addition, the bar code labelling of blood product information by the transfusion service, together with the availability of reliable bar code readers, are important factors in creating a climate wherein the introduction of data processing now becomes appropriate.

Accepted for publication 20 April 1984
In late 1981 the computer section of the Wolfson Research Laboratories undertook to design and develop a pilot data processing system providing for routine clerical, patient record, and stock management aspects of blood bank laboratory work. The pilot system was to be installed and field tested at Good Hope Hospital, Sutton Coldfield, West Midlands. In order to ensure general applicability of the proposed package, a working party comprising senior representatives of the Regional Blood Transfusion Service, consultant haematologists, and medical laboratory scientific officers actively involved in blood banking was formed to meet regularly with Wolfson Research Laboratory computer staff.

The end user objectives of the system were defined as:

1 To reduce clerical involvement by laboratory staff at all stages in the preparation and retrieval of laboratory records.

2 To improve legibility and reduce transcription errors in labels, reports, and records.

3 To provide additional checking facilities at critical stages in the selection and issuing of products for transfusion purposes.

4 To improve stock management and allocation procedures. 
The system design objectives were defined as:

1 Table and parameter driven software facilitating implementation in differing laboratories.

2 Implementation on low cost hardware as a stand alone multiuser system.

3 Portability - that is, implementable on a family of processors of varying capacity and power, either as a stand alone system or possibly on a shared or bureau basis.

4 Inclusion of light pens as an integral part of the system hardware enabling automatic input of bar coded blood product information.

The second and third system design objectives were at the time (late 1981) the most difficult to meet and it was finally decided that MUMPS provided the most flexible in terms of hardware and software.

MUMPS (Massachusetts Universal MultiProcessing System) ${ }^{1}$ is an interactive multiuser data management system, heavily oriented towards the handling and storage of textual rather than numerical data. Although MUMPS is usually referred to as a language, it is a combination of operating system, language interpreter, and database manager. The operating system is largely transparent to the user; the language approximates to a terse form of BASIC and the database manager effectively allows the user to consider both memory and disk as content addressable storage systems rather than the position addressable concept associated with most other languages. It is this last feature which gives MUMPS great power and flexibility when dealing with cumulative laboratory records of extremely variable length, indexed by several widely differing alphanumeric keys (for example, surname, registration number) and also provides for efficient packing and therefore greater storage of data.

MUMPS provides an interpretive language which facilitates rapid program development and modification. As with many clinical laboratory applications blood bank data processing contains a high component of terminal input; thus the reduced computational speed of interpretive languages is not a serious limitation especially as the MUMPS operating system gives priority to input/output transactions thus optimising response at terminals.

Other advantages of this approach accrued from the existence of a MUMPS based clinical laboratory package, developed by Trent Regional Health Authority from a MUMPS version of PHOENIX, ${ }^{2-4}$ which provided a suitable skeleton on which to develop the proposed system.

The Digital Equipment Corporation (DEC) Micro 11 system was chosen as the target hardware. Because of delivery and other problems, however, development and evaluation has been based on a
PDP 11/23 system lent to the Wolfson Research Laboratories by Digital Equipment Corporation.

The blood bank laboratory software can be run on any of the DEC family of computers capable of running MUMPS under a DSM-11 operating system, version 2.1 or higher, with a minimum 8 kilobytes partition size. The minimum hardware configuration must include:

1 Computer capable of running MUMPS under DSM-11

2 Disk drive - minimum capacity 5 megabytes

3 Visual display unit

4 Eighty column character printer

A number of blood transfusion centres in the UK use labels which include bar coded blood product details, ${ }^{5}$ the code adopted being stardardised as Codabar. ${ }^{6}$ In order to make entry of stock information as rapid and as accurate as possible, the system has been designed to allow bar code input of such details where these are available.

The bar code readers employed (manufactured by Intermec) operate in line with the RS232 serial link between the processor and each visual display unit. The computer receives data generated by the reader on the same channel as that from the keyboard, so that no additional interfacing and minimal additional software are required to implement bar code input.

Facilities provided by the package may conveniently be described under three main headings: $(a)$ system definition; (b) stock management; and $(c)$ request management.

\section{System definition}

The flexibility afforded by the system's table and parameter driven software requires new users to specify numerous details of required operation within their environment. Considerable thought must be given to this process before attempting to set up a system via the interactive visual display unit routines provided.

The more important parameters that must be defined to the system are now described.

\section{PATIENT DETAILS}

Data required at patient request entry are defined from a library of possible prompts. All or a selection of the following information may be collected and retained for each patient. Case number and surname fields are automatically included as the first and second prompts of the request screen - this necessity reflects the basic structure of the patient record system. 
1 Case number

2 Surname

3 Forename

4 Maiden name

5 Sex

6 Date of birth

*7 Location

8 Provisional blood group

9 Confirmed blood group
* 10 Hospital

*11 Consultant

*12 Surgical procedure

*13 Diagnosis

14 Ethnic group

15 Special interest flag

16 Antibody identity

17 Kleihauer flag

18 Patient address

Optimal prompts (3-18) can be included in any order and may be included or removed at any time during the life of the system.

All or a selection of the asterisked fields may be specified for retention for each patient request made. If none are specified, then each time a new request is made for a patient, changes entered for these fields will replace the original and information will be lost. By electing to retain some or all of this data the contents of the field are "attached" to the request record and made available at subsequent points in the system.

\section{STOCK DETAILS}

Similarly, stock entry parameters must be defined.

1 Unit number

2 Product code

3 Blood group

4 Expiry date

5 Entry date
6 Additional blood group(s)

7 Blood transfusion service issue note reference number

8 Location of unit in laboratory

9 Source of unit

10 Comment relating to the unit of blood

Unit number, product code, blood group, expiry date are mandatory fields. Optional items $(6-10)$ may be included in any order and altered at will.

\section{CODE DIRECTORIES}

Directories allowing coded entry for many of the patient request and stock related fields may be defined.

$\begin{array}{ll}\text { *Product code } & \begin{array}{l}\text { Diagnosis } \\ \text { *Blood group + Rh factor } \\ \text { Ethnic group } \\ \text { Stock location }\end{array} \\ \begin{array}{l}\text { *Interest flag } \\ \text { Stock comment }\end{array} & \text { Hospital code } \\ \text { Stock source } & \text { Patient location } \\ \text { Method of disposal } & { }^{*} \text { Antibody identity } \\ \text { Consultant/general } & \text { Report comment } \\ \quad \text { practitioner code } & \text { Specimen comment } \\ { }^{*} \text { Qualitatives result codes } & { }^{*} \text { Technician initial }\end{array}$

Surgical procedure

With the exception of the asterisked fields data may be input optionally as free text. It is recommended that codes are set up where possible to allow greater speed of entry, more efficient data storage, and statistical analysis of workload.

A text expansion generally of $20-30$ characters

must be associated with each code defined. Optionally, a bar code number may also be associated with each code input, allowing codes to be read from a bar coded menu of possible codes.

Product code and blood group codes should correspond to those used by the issuing centre, so that bar coded labels affixed to blood products may be read into the system.

\section{TEST DEFINITION}

Tests, defined as single assays producing single qualitative results, must be identified to the system as a test code or mnemonic and test name. Additional information is required for tests which constitute part of a group or crossmatch operation. For the former the expected result for each of 15 possible blood groups must be specified and for the latter the expected crossmatch result declared.

\section{SET DEFINITION}

Tests, once defined to the system, are grouped together into test sets. A test set comprises tests (or a single test) which are always requested together and which must be examined together to produce new parameters such as blood group. Sets are identified principally in terms of set code or mnemonic, set name, specimen type, and reporting group. The latter parameter defines combinations of completed test sets required (if requested) before a report can be produced. Additionally, tests within test sets must be linked with worksheet codes; all tests within a set may appear on the same worksheet or may (except for group and crossmatch tests) be distributed to several. Test sets (up to a maximum of six) may be grouped together into "supersets" to facilitate request entry. For example, group and crossmatch requests may be entered via a single superset code rather than two set codes.

\section{WORKSHEET/LISTS}

Users are required to specify worksheet structures characterising batches in which work will be processed. A maximum batch size must also be specified. Quality control samples may be specified for automatic inclusion at specified points within worksheet batches. Elections made at this stage may be altered at worksheet generation to meet actual or emergency requirements, or both. For each quality control sample defined for a worksheet a quality control reference number and name must be defined together with an expected result $(+$ or - ) for each test included. If no quality control check is required as part of result authorisation for a particular test, then this may be specified.

A series of flags is used to specify aspects of system operation-for example, (a) print formats for 
worksheets, "day" books, etc; and (b) batch or single step authorisation for grouping, crossmatching, and other results.

\section{OTHER STOCK PARAMETERS}

Additional stock related parameters requiring definition relate to products that may be issued without recourse to crossmatch procedures and the definition of optimum stock levels. This latter definition is required to allow the system to create stock reorder lists on demand.

\section{CULLING/STATISTICS}

The final part of system tailoring is concerned with system housekeeping, where elections made must reflect the amount of on line disk storage available.

Current indications are that a laboratory servicing 8500 requests a year, receiving 8200 donor units per year, and performing an average of 2.4 crossmatches per unit uses 0.8 megabytes of disk storage per month to retain all patient and stock data. Consequently one year's data can be retained on line for laboratories of this type on the most common unit of disk storage - 10 megabytes exchangeable or fixed Winchester technology available as standard on all lower end DEC PDP 11 series hardware.

The length of time that patient data (including results) and stock data are retained must be defined and specific patient subpopulations identified for longer or shorter retention. Similarly, subpopulations for which statistical data accumulation is required must be specified. If these are not set up then only general monthly statistics will be accumulated - that is, the number of requests and test sets processed, the number of units processed, the number of units crossmatched, and so on. In both cases subpopulations may be identified on the basis of blood group, consultant, general practitioner, surgical procedure, diagnosis, location, and special interest flag.

The time required accurately to define laboratory practices, protocols, and so on to the system should not be underestimated. It is an unavoidable overhead accruing to flexible generalised software packages. It is unlikely, however, that a laboratory would ever need to repeat the exercise completely once it was successfully accomplished, and users may be comforted by the prospect that it is unlikely that future changes, modifications, and extensions to laboratory practice could not be met by redefining parts of the system.

\section{Stock management}

If the system is to be effective in the management of resources it is essential that each unit of stock enter- ing the laboratory is booked into the computer system. Conversely, each unit that is transfused, returned to the issuing centre, or disposed of in any other way must be removed from the system.

Information entered against each unit of stock depends on decisions made during system definition. Unit number, product code, blood group, expiry date, and entry date are essential items for each unit and may, with the exception of expiry date, be entered via bar code readers virtually from the crates in which the stock is delivered. Special facilities have been provided to enable replication of data occurring in batch deliveries.

In addition to data entered on arrival in the laboratory, various other items of information are retained or updated by the system, or both, as a byproduct of routine daily operation. Information retained for each unit includes:

The number of preliminary and confirmed reservations made.

Date of last confirmed reservation.

Laboratory number of current reservation.

Current reservation status (that is, FREE, RESERVED, etc)

Date used (if appropriate).

Disposal code, where relevant.

Diagnosis code if the unit was allocated to a patient.

Surgical procedure if the unit was allocated to a patient.

Consultant code if the unit was allocated to a patient.

Patient identity if the unit was transfused.

These data provide the basis for resource management and product accountability. Sorted or selected information, or both, may be listed at any time in a variety of ways including:

Stock breakdown by location and any combination of product type, blood group, and reservation status.

Stock level synopsis and stock reorder lists.

Identification by location of expired stock and related stock management procedures-for example, entry of disposal codes.

Stock usage statistics-breakdown by month to aid the preparation of DHSS statistical returns.

\section{Request management}

Information relating to the identity of the patient together with details of the specimen and tests/ products required must be supplied to the system before any data processing associated with routine laboratory work is attempted. 


\section{REQUEST ENTRY}

Each patient is distinguished from all others by means of a unique case number, usually the hospital registration number. Where no such number is available from an external source, the system will itself allocate a unique number having a $\mathrm{Z}$ prefix. The system creates and maintains a master index of patients or, if run in conjunction with the Trent Regional Health Authority version of Phoenix MUMPS, could access its patient master index. If a case number occurs in the master index and the first two characters of the surname input agree with those in the master index, previously entered patient demographic data will be displayed. Where the case number is not known a name search can be initiated, which allows the user to select the appropriate patient from a list of possibilities. A new entry to the master index may be made by inputting a new case number together with all relevant patient details.

Details of the specimen, together with work required, are entered in terms of:

Laboratory number Date/time requested

${ }^{*}$ Product requested Work required (as test sets)

*Quantity required Specimen comment

${ }^{*}$ Date/time required

Asterisked fields are required only for crossmatch, issue, or reissue requests.

When a specimen arrives in the laboratory it must be allocated a unique laboratory or specimen number of the general form Y.NNNN.X where:

$$
\mathrm{Y}=\text { last digit of the current year }
$$

NNNN. $=$ a number in the range 1 to 9999

$$
\mathrm{X}=\text { check digit (modulus } 11 \text { ) }
$$

Laboratory numbers may be bar coded, in which case identical labels may be affixed to the request document and specimen container. Where numbers are keyed into the system the following alternative formats are acceptable:

NNNN. The current year will be assumed and the check digit calculated. Leading zeros may be omitted.

Y.NNNN. Again the check digit is calculated. On entry of a laboratory number specimen details will be displayed if the number is already known by the system and is associated with the patient. On first entering a unique laboratory number to the system, however, the user must enter some/all of the above details.

Request details may be input as request forms are received in the laboratory or may be batched for subsequent entry. It is essential, however, that full details be entered before any attempt is made to enter results or to allocate blood products.

Work that has been requested but has not yet been allocated to a worksheet is considered by the system to be outstanding work. The user may list outstanding work for all or selected work areas. The list(s) produced will be ordered on the basis of the date and time products are required and will contain the name of the patient, the date and time the request was made, and other test sets that have been requested, together with their relevant status - that is, outstanding $(\mathrm{O})$, worksheeted $(\mathrm{W})$, or reportable (R).

WORKSHEET/LIST GENERATION

Outstanding work may be allocated to different work areas within the laboratory in a variety of ways, facilitating throughput for both routine and emergency specimens. Worksheets may be generated in one of a number of possible hardcopy formats or as softcopy at visual display units and may be in order of the date and time the requested product is required-a particularly useful feature in the management of crossmatch requests.

The ability to obtain information on requests received but not yet processed through the laboratory is complemented by the ability to list work already allocated to various work areas and by inquiry procedures, oriented to patient case number and laboratory number, which are described later.

\section{PRELIMINARY RESERVATION OF BLOOD} PRODUCTS

The allocation of a request to a worksheet signals the start of request processing both in the analytical and data processing sense. Where blood products are to be reserved against a request, the user may either do this at the conclusion of the worksheet allocation stage (in which case relevant unit numbers will appear on crossmatch worksheets), or may delay this preliminary reservation until a later stage. If this latter course is taken then crossmatch worksheets will merely refer to bag numbers $1-\mathrm{N}$ depending on number of units requested. Irrespective of the stage that a preliminary reservation is made, the user may reserve more or fewer units than originally requested.

To effect a preliminary reservation the unit number of the product is entered, preferably by reading the bar coded unit number label, although keyboard entry is allowed. The system displays all available products with this unit number and the user must select the one to be reserved. A product will not be reserved by the system if it is already reserved for another patient or will expire before the date it is required for use.

The product type and the blood group of the unit reserved are compared with the product type requested and the patient blood group, respectively. A warning message is displayed if any discrepancies 
are found and the user is given the choice of accepting or rejecting the unit.

The reservation period is normally set to two days after the date required. This may be changed and a unit can be reserved for a maximum of 99 days from the date required.

A list of units previously crossmatched for a particular patient may be requested. The units listed will be those where a reservation was confirmed and the unit subsequently returned to stock. The user may choose to list only those units still available for reservation.

\section{RESULT ENTRY}

Qualitative data, which may only be expressed as + , -, or multiples of the former, are keyed into the system in the sequence defined by the relevant worksheet/list. Entry may be by row-that is, all results for a particular sample, which may be patient or donor-or by column-that is, all results for a particular test. In either mode only the test result actually being input is displayed in order to prevent previously entered results influencing the operator. But patient identity, laboratory number, and other relevant details associated with the data being input are also shown. Results may be edited and comments added at several subsequent stages. Comments may be code or free text, or both, and may be designated for laboratory use only-that is, not to appear on report documents.

\section{AUTHORISATION}

Before the generation of patient reports and product labels all result data must be "authorised,"-that is, certified as checked. This may be done only by laboratory staff previously identified to the system as "approved" authorisers. The initials of people authorising results are permanently associated with each set of results within each patient record.

An underlying principle adopted throughout the system development has been that the laboratory worker should not be denied the opportunity to exercise judgement in the prosecution of his or her job. It was considered to be in the interests of all, however, to provide the ability to monitor some aspects of user interaction and to leave it to the discretion of the laboratory director to establish and maintain certain procedures within the laboratory. Essentially, particularly sensitive decisions such as authorisation of products not of the type requested or not of the same blood group as the patient, changes to stock, and patients' details or results are automatically recorded on a checklist, which may be printed at some convenient time and retained as part of the laboratory's records.

Authorisation is organised on a worksheet/list basis and should not be attempted until laboratory staff are satisfied that all relevant results have been entered for all specimens allocated to a particular worksheet. A basic precept of the system is that a worksheet/list represents a regular matrix of results, all of which are required to be provided-that is, all tests must be performed and entered for every patient, control, and donor sample included on any worksheet. A further precept of the system is that results once authorised may not be changed. Consequently, authorisation should be viewed as a critical stage in routine data processing faeilitating the monitoring of data and products leaving the laboratory.

Essentially, authorisation entails:

1 For "group" tests - the automatic derivation of blood group from test results entered and where appropriate the comparison of this derived result against available preliminary or confirmed patient blood group

2 For "crossmatch" test sets—automatic checking of crossmatch compatibility

3 Association by the authoriser, where necessary, of coded or free text comments, or both

4 Transfer of authorised results from work files to patient files and marking them with the authoriser's initials

5 Creation of report indices thus permitting the generation of reports and crossmatch labels.

Results may be authorised in single step or bata. mode. In single step mode, patient details and results are displayed on a formatted screen and authorisation is performed at the user's discretion. Batch mode assumes that the results have already been visually inspected and that the results are to be authorised without display or user intervention.

In either mode authorisation will fail if:

1 The group or crossmatch pattern match fails

2 Test set results for a patient or control, or both, are incomplete

3 A quality control test fails

4 An apparently inappropriate product has been reserved for a crossmatch request, or if no product has been reserved.

When authorisation fails, all relevant patient/ specimen details together with the reason for failure are displayyed (Figs. 1 and 2) and the user must then decide which course of action to pursue. For exam- N ple, where the system has failed to etablish a blood $N$ group from the results entered, the user may make N his or her own selection, which will then be checked $\omega$ against any existing value of confirmed or preliminary blood group for that patient. If there is $a$ conflict a warning message will be issued; if this or any other warning message is overridden all relevant details will be logged on to the system checklist and 


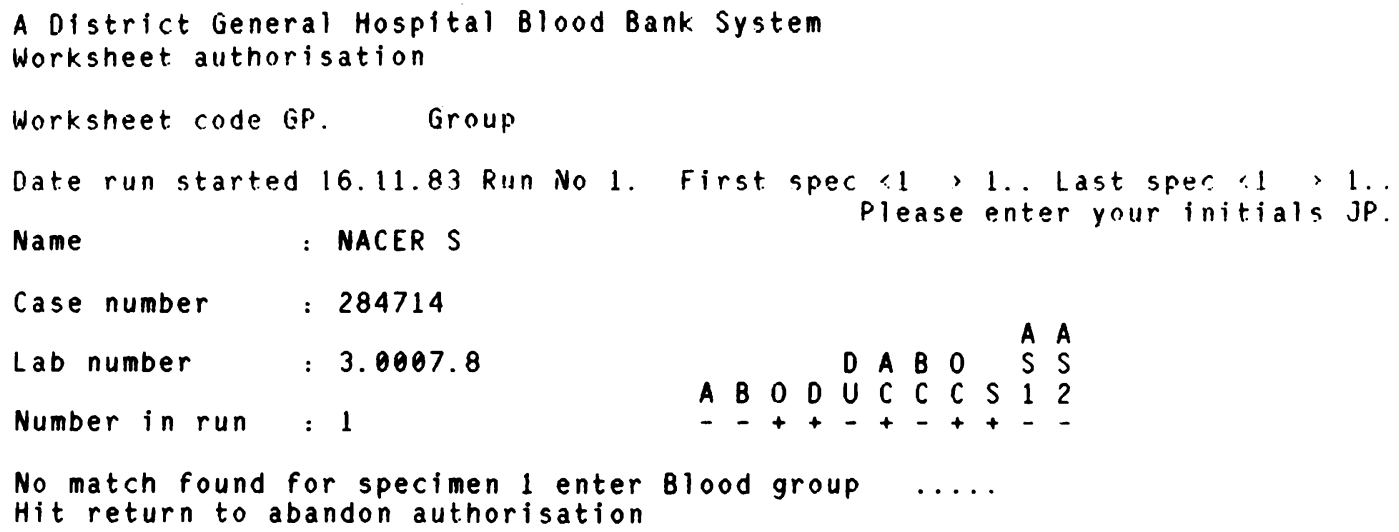

Fig. 1 Screen display during authorisation of unrecognisable blood group results.

subsequently generated (if desired) as a permanent hardcopy record. Alternatively, the user may decide to abandon authorisation and go back and reanalyse/amend results. If authorisation detects that an incompatible or inappropriate product has been reserved then again the user may choose to override the warnings given. Compatible or incompatible units may be returned to stock at this stage; the former, however, only if single step authorisation has been specified.

If a test set is incomplete the user must abandon authorisation and return to result entry.

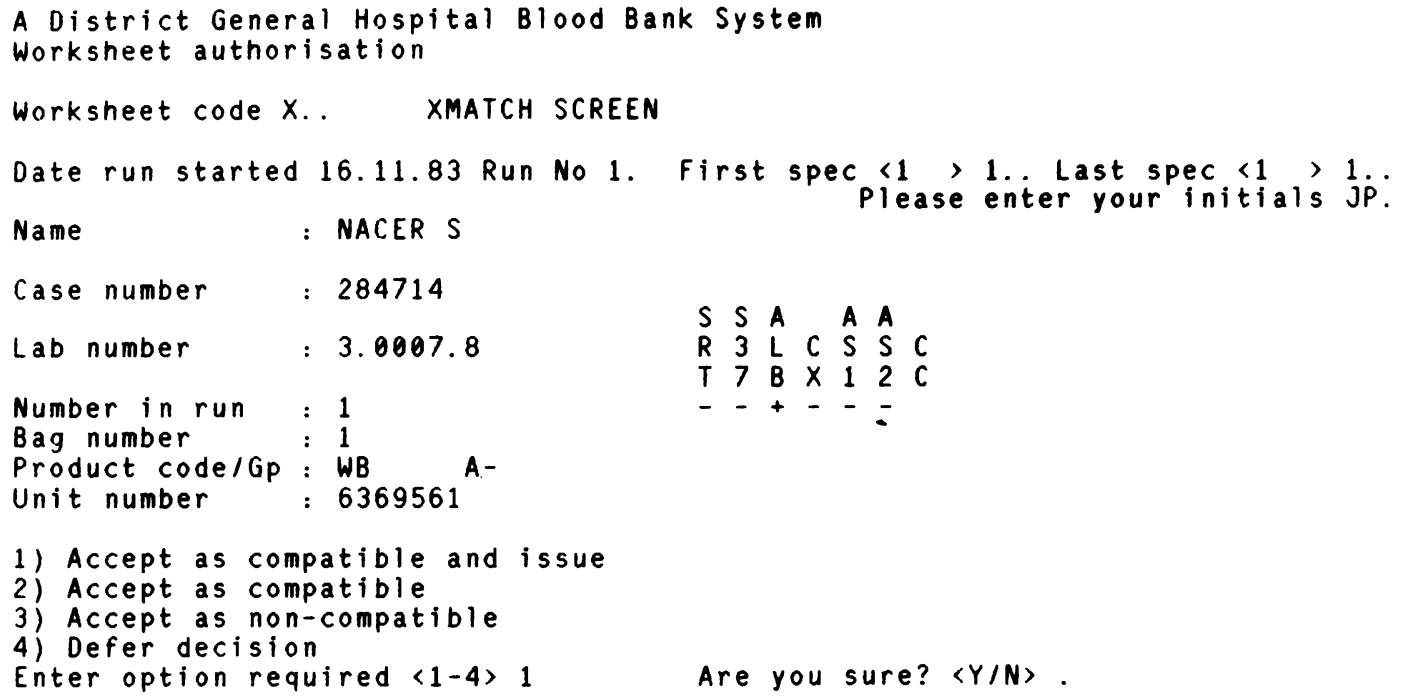

Fig. 2 Screen display during authorisation of crossmatch results indicating incompatibility. 


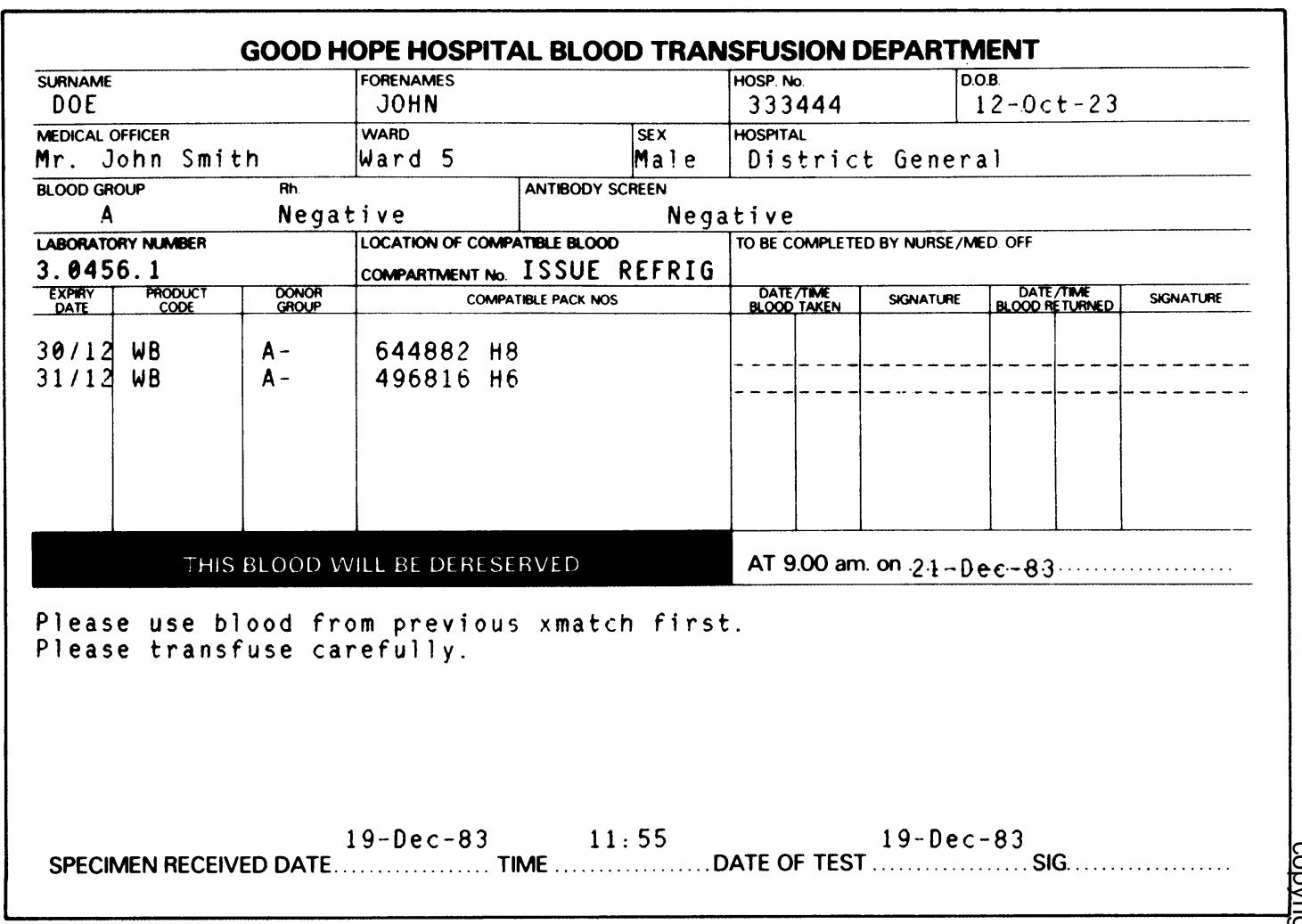

Fig. 3 Patient report.

Where there is a quality control failure the user may override the warning message or may cancel the whole or part of the relevant worksheet and reprocess the specimens in question.

\section{REPORTS AND CROSSMATCH LABELS}

Reports and crossmatch labels (Fig. 3 and 4) for authorised data may be generated at any time, provided that all sets specified for simultaneous report-

\begin{tabular}{|c|c|c|c|}
\hline \multicolumn{2}{|c|}{ COMPATIBLE WITH } & \multicolumn{2}{|c|}{ COMPATIBLE WITH } \\
\hline \multicolumn{2}{|c|}{$\begin{array}{l}\text { DONOR UNIT No. } \\
644882 \mathrm{H8}\end{array}$} & \multicolumn{2}{|c|}{$\begin{array}{l}\text { DONOR UNIT No. } \\
496816 \text { H6 }\end{array}$} \\
\hline \multicolumn{2}{|c|}{$\begin{array}{l}\text { Surname. } \\
D O E\end{array}$} & \multicolumn{2}{|c|}{$\begin{array}{l}\text { Surname } \\
D O E\end{array}$} \\
\hline \multicolumn{2}{|c|}{$\begin{array}{l}\text { Forenames } \\
\text { JOHN }\end{array}$} & \multicolumn{2}{|c|}{$\begin{array}{l}\text { Forenames. } \\
\text { JOHN }\end{array}$} \\
\hline \multicolumn{2}{|c|}{$\begin{array}{l}\text { Reg. No. } \\
333444\end{array}$} & \multicolumn{2}{|c|}{$\begin{array}{l}\text { Reg. No. } \\
333444\end{array}$} \\
\hline 5 & $\begin{array}{l}\text { Hospital } \\
\text { DGH }\end{array}$ & $\begin{array}{l}\text { Ward } \\
5\end{array}$ & $\begin{array}{l}\text { Hospital } \\
\text { DGH }\end{array}$ \\
\hline \multicolumn{2}{|c|}{ Date of Birth $12-0 c t-23$} & \multicolumn{2}{|c|}{ Date of Birth $12-0 \mathrm{ct}-23$} \\
\hline \multicolumn{2}{|c|}{ COOD HOPE BLOOD BANK } & \multicolumn{2}{|c|}{ COOD HOPE BLOOD BANK } \\
\hline
\end{tabular}

Fig. 4 Compatibility labels. ing are available. Generalised report and label generators are not included in the system software as the complications of such routines would conflict with the relative simplicity of providing customised formats. Reports and labels may be generated on the basis of specific case numbers or of results authorised since the last reporting run. Any recent report or label.may be reprinted if required.

\section{INQUIRIES}

Telephone and other inquiries can be serviced by comprehensive inquiry facilities accessed either on the basis of patient case number, name, or laboratory number. Where the patient case number or name is used, all or specific parts of the accumulated data may be displayed or obtained as hardcopy. Specification may be on the basis of test set or dates of requests, or both. Where laboratory number is used then only organisational data relating to the management of a particular request can be obtained-for example, sets requested together with their status (worksheeted, authorised, reportable, etc).

A hardcopy record of laboratory activity-that is, 
all requests logged and reports generated within a specified time period-may be obtained in a variety of formats either by patient name or laboratory number, or both. If the time period is specified as one day then the "laboratory day book" may be automatically obtained.

Workload statistics may be obtained on demand. The overall breakdown is by month and includes the number of requests processed and details of sets requested.

\section{Additional facilities}

Other request management facilities provided by the system include various important manipulative functions providing for the less routine aspects of blood bank work.

Previously crossmatched units, if still available, may be reissued without retesting - in other words, such units may be rereserved and reauthorised ready for report and label production in a single operation.

The period of time for which product(s) are reserved against a particular request may be extended, after authorisation, to meet changed circumstances. Also additional comments may be added to authorised results; authorised results themselves, however, may never be altered.

A record of isolated antibodies may be maintained for any patient on the system. On entry of the patient case number antibody data may be inspected, added, or deleted. These data are made available at several points in the system-for example, request entry, product reservation, inquiries.

The various facilities provided by the system are accessed via a series of menus. User definable log in codes allow access to different families of menus; this password concept is designed primarily to separate frequently used routine functions from those used mainly at implementation time, rather than as a security system to prevent unauthorised use. As passwords are not displayed on input and must be allocated during the installation phase, however, it is possible to preclude access to some areas of the system.

The system software is largely self documenting in that informative text may be invoked at the visual display unit at almost every point where input to the system is required. Additionally, code directories may be displayed at relevant points. On call staff and other less frequent users therefore have at their fingertips a substantial quantity of information facilitating their use of the system. A user manual is also available in which emphasis has been placed on guiding the user to tailor the package to individual requirements.

\section{Conclusion}

The software package that has been developed provides a data processing environment designed to meet end user objectives specified for the system. The degree to which these objectives are attained in differing laboratories introducing this system will depend ultimately on the acceptability and scope of its overall design.

Portability has been shown by implementing the package on a DEC $11 / 44$, an $11 / 23$ and a Professional 350 , the latter running under "Standard Micro MUMPS" supplied by Structured Data Systems. Implementation costs for stand alone systems range between about $£ 9000$ for two user system with two printers based on a Professional, and $£ 18000$ for a five user, three printer system based on an $11 / 23$.

A system running on a DEC $11 / 23$ with a 256 kilobyte memory, twin 10 megabyte disks, three visual display units, two light pens, and two printers was installed at the Blood Bank at Good Hope Hospital, Sutton Coldfield, West Midlands in May 1983. The department handles 8500 patient requests and 8200 donor units annually.

The application software was commissioned in two days and staff training was completed within a week. Use of the equipment has transformed the pattern of work within the department, but all staff have adapted well to the changes and staff acceptability of the equipment has been high.

Considerable time has been saved with many operations. One hundred units may be booked into the system in only three minutes, eliminating a substantial amount of clerical work. Similarly, the dereservation of blood can be performed extremely rapidly, saving up to $90 \mathrm{~min}$ a day. Initially, group and crossmatch procedures took much the same time as with the former techniques, but as expertise increased the time taken has now decreased slightly. The worksheet principle is ideally suited to the handling of batches of 10 or more samples, but the need to generate one or more worksheets for a single sample, especially if it is urgent, seems to be unduly time consuming. Modifications to the software to speed up the handling of urgent samples are under review.

A major benefit of the system has been the production of extremely legible report forms and labels, which have been well received by the clinicians. The handling of inquiries has been greatly eased and accelerated by use of the computer, an improvement much appreciated throughout the hospital. The elimination of hand written records with the attendant risks of transcription errors, and the many safeguards within the system, have combined to 
reduce the possibility of a serious human error. This unseen benefit is one of the most important advantages to be gained from the use of the new equipment.

It has not yet been possible to assess the ability of the system to provide records of blood product usage to the Regional Transfusion Centre, but within the hospital department the generation of statistics has been streamlined. It has been possible to assemble information regarding the request and actual usage rates for blood products in connection with particular surgical procedures, and this information may prove valuable in influencing changes in request patterns. It is too soon to establish whether the use of the equipment has reduced the wastage of blood. All other previously defined objectives have, however, been achieved. The hardware and software have proved reliable and the system appears to be sufficiently flexible to be used in a wide range of hospital laboratories.

Since initiation of the project, an additional requirement has arisen for further accountability for blood product usage and transfer to other hospitals. The information required is available within the system and modification of output to a form suitable for transfer to the Blood Transfusion Service centre as outlined in the DHSS draft Health Circular BLD/ $2 / 13$, is under review.
We thank the following groups for their assistance throughout the project: Digital Equipment Corporation, Trent Regional Health Authority, Laboratory Computer Team, and the West Midlands Region Blood Bank Working Party.

The financial support of the Department of Health and Social Security is gratefully acknowledged.

\section{References}

MUMPS Development committee, American National Standard MUMPS Language Standard: American National Standards Institute ANSI X11.1, 1977.

${ }^{2}$ Abson J, Prall A, Wootton IDP. Data processing in pathology laboratories: The Phoenix system. 1. The overall design and the main facilities. Ann Clin Biochem 1977; 14:307-14.

'Abson J, Prall A, Wootton IDP. Data processing in pathology laboratories: The Phoenix system. 2. The file structure and basic operations. Ann Clin Biochem 1977;14:315-22.

${ }^{4}$ Abson J, Prall A, Wootton IDP. Data processing in pathology laboratories: The Phoenix system. 3. Further programs, staff training and system performance. Ann Clin Biochem 1977; 14:323-9.

5 Ibbotson RN, Jackson RE. Data capture by the use of bar-coding in a blood transfusion centre. Med Lab Sci 1980:37:237-42.

- Committee for Commonality in Blood Banking Automation. The $C C B B A$ labeling system and code assignments for blood products. Arlington, Virginia: American Blood Commission 1977 